\section{$\underset{\substack{\text { hommes } \\ \text { \& migrations }}}{ }$}

\section{Hommes \& migrations}

Revue française de référence sur les dynamiques

migratoires

$1310 \mid 2015$

Fashion Mix

\title{
La confection et les immigrés à Paris
}

Une histoire ancienne

Nancy L. Green

\section{(2) OpenEdition}

1 Journals

\section{Édition électronique}

URL : http://journals.openedition.org/hommesmigrations/3140

DOI : 10.4000/hommesmigrations.3140

ISSN : 2262-3353

Éditeur

Musée national de l'histoire de l'immigration

\section{Édition imprimée}

Date de publication : 1 avril 2015

Pagination : 7-12

ISBN : 978-2-919040-31-5

ISSN : 1142-852X

\section{Référence électronique}

Nancy L. Green, «La confection et les immigrés à Paris », Hommes \& migrations [En ligne], 1310 | 2015, mis en ligne le 01 avril 2018, consulté le 30 avril 2019. URL : http://journals.openedition.org/

hommesmigrations/3140; DOI : 10.4000/hommesmigrations.3140 


\section{LA CONFECTION ET LES IMMIGRÉS À PARIS UNE HISTOIRE ANCIENNE}

Par NANCY L. GREEN, directrice d'études, École des hautes études en sciences sociales.

La mode parisienne a besoin des immigrés. Mieux, son développement accéléré depuis le milieu du XIXe siècle n'aurait pu se faire sans eux. Le secteur de la confection traverse des pénuries de main-d'œuvre chroniques. II recrute par conséquent des étrangers qui arrivent à Paris et qui cherchent du travail. Juifs de tous horizons, Polonais, Russes, Belges, Arméniens ou Chinois, les migrants continuent de prendre part à la fabrication et au commerce de vêtements, contribuant à la renommée de la capitale mondiale de la mode.

Comment parler de la "mode en France" et des étrangers sans parler des travailleurs immigrés de la confection ? La mode est en grande partie fabriquée par eux, et cela depuis plus d'un siècle et demi. La mode est "fashion", certes, mais elle est produite pour toutes les bourses. La mode "d'en bas", voire "basic" et à bas prix, c'est la mode de tous les jours. C'est aussi un mode de production, où les grands couturiers ont toute leur place, les petites mains et les mécaniciennes de la confection également.

La confection de vêtements en série est une industrie qui s'accroît depuis le XIX siècle grâce à une grande "découverte" : les tailles humaines se ressemblent. Cette fabrication de vêtements a commencé par des uniformes pour militaires et des articles pour les colonies dès le XIX ${ }^{e}$ siècle. Les hommes en étaient les premiers destinataires. Ce type de fabrication va s'étendre progressivement à l'habillement féminin. Dans un célèbre article de 1904, Georg Simmel décrit la mode comme étant une expression à la fois d'uniformité et d'originalité ; il s'agit de se distinguer des autres tout en portant des signes qui permettent de s'identifier au sein de son groupe d'appartenance ${ }^{1}$. Ce paradoxe qui lie différenciation et similitude peut être considéré comme un fondement de l'industrie de la confection, tiraillée entre art et industrie, entre 
flexibilité et standardisation. Le climat et le genre participent également à la création de la mode. Les saisons sont inscrites dans la fabrication des cols roulés pour l'hiver et des maillots de bain pour l'été. Depuis "la grande renonciation masculine" au début du XIX siècle, quand les hommes ont délaissé leurs perruques et leurs plumes pour les costumes-cravates, laissant la variation de couleurs et de formes aux femmes ${ }^{2}$, ce sont les vêtements féminins qui ont dû fabriquer la différence dans la similitude. Voilà tout un programme philosophique qui sera le défi de la confection. Et qui sera incarné pour une grande partie dans les ateliers d'immigrés.

\section{Au service de la flexibilité}

"Flexibilité", ce nom est inscrit dans l'industrie de l'habillement comme la forme de fonctionnement d'un système tiré entre standardisation et différentiation. Il s'agit non seulement d'une flexibilité pour décider quand lancer plus de bleu ou plus de jaune, mais d'un mode d'organisation de la fabrication elle-même. La sous-traitance va devenir un mode d'organisation qui, jusqu'à nos jours, favorise les immigrés dans un double sens. Il faut relativement peu de capital pour ouvrir un atelier de confection. Et c'est au sein de telles structures qu'immigrés et surtout immigrées peuvent se mettre au travail rapidement, au sein d'ateliers de compatriotes, où l'on peut travailler entre soi, dans sa langue, chômant ensemble au moment des fêtes. Petits prix de la mode, petits prix pour les ouvriers et ouvrières de la mode. Les bas salaires et les conditions très dures - surtout pendant la saison - sont le fléau des ouvriers, mais la condition de la mise en marche de ces ateliers. En somme, la flexibilité requise pour répondre aux demandes de saisons et de couleurs, la structure de la fabrication en sous-traitance et dans de petits ateliers, les bas coûts d'entrée dans le métier, à la fois en capital et en savoir-faire, tout se conjugue avec l'arrivée d'immigrés pour fabriquer la mode faite en France ${ }^{3}$.

Si la structure y est propice, on peut s'interroger pour les confectionneurs comme pour les couturiers étrangers : importent-ils la mode ou la créent-ils sur place? Dans les années 1840, environ $40 \%$ des tailleurs parisiens étaient étrangers, originaires d'Allemagne, de Hongrie et de Pologne, bien que la plupart de leurs ouvrières fussent françaises ${ }^{4}$. À la fin du XIX siècle, l'essor d'une mode de vêtements de sport féminins et une certaine masculinisation de la mode féminine ${ }^{5}$ coïncident avec l'arrivée d'immigrés de Hongrie, de Russie, de Bohême-Moravie ou d'Allemagne, où les hommes avaient toujours travaillé dans le vêtement féminin. On estimait qualors $85 \%$ des travailleurs du vêtement pour femmes étaient des étrangers (comparé à $35 \%$ dans la confection masculine) ${ }^{6}$. Certaines modes sont clairement influencées par les immigrés eux-mêmes. Les juifs russes, les Allemands, les Hongrois et les Suédois constituent environ 80 \% de la main-d'œuvre dans le secteur de la fourrure, qui va doubler son chiffre d'affaires entre 1870 et $1895^{7}$. Sans parler des imperméables, autrefois importés d'Angleterre mais désormais fabriqués à Paris par les immigrés d'Europe centrale et orientale. Enfin, la popularisation de nouveaux sports telles l'automobile et la bicyclette à la fin du XIX siècle va de pair avec une nouveauté attribuée aux immigrés juifs : la casquette à la russe (plate) fait fureur, qu'elle soit bourgeoise ou ouvrière, et les immigrés ont permis à ce sec-

2. John Carl Flügel, The Psychology of Clothes [1930], New York, AMS Press, 1976, pp.110-117; Anne Hollander, Sex and Suits, New York, Knopf, 1994. 3. Nancy L. Green, Du Sentier à la 7 e Avenue. La Confection et les immigrés, Paris-New York (1880-1980), Paris, Seuil, 1998 ; Solange Montagné-Villette, Le Sentier. Un espace ambigu, Paris, Masson, 1990 ; Maurizio Lazzarato, Yann Moulier-Boutang, Antonio Negri, Giancarlo Santilli, Des entreprises pas comme les autres. Benneton en Italie, le Sentier à Paris, Paris, Publisud, 1993. 4. Louis Chevalier, La Formation de la population parisienne au XIXe siècle, Ined, Travaux et documents, $n^{\circ} 10, P a r i s, ~ P U F, 1950$, p.182 ; François Faraut, Histoire de la Belle Jardinière, Paris, Belin, 1987, pp. 28-29. 5. Nancy L. Green, "Des tailleurs et du tailleur. La masculinisation de la mode féminine”, in Guillaume Erner, Mode, où est ta victoire?, Paris, éd. de l'Herne, 2013, pp. 251-259. 6. L'Humanité, 22 juillet 1913 ; il s'agit d'une estimation du_syndicaliste Pierre Dumas, qui s'inquiétait du flux de nouveaux ouvriers et qui a peut-être exagéré leur importance. 7. Pierre du Maroussem, La Petite Industrie (salaires et durée du travail), vol. 2, Le Vêtement à Paris, Paris, Imprimerie nationale, 1896, p. 702. 
teur importateur (30 à 40000 casquettes par an) de se stabiliser et de plus que doubler ses exportations (jusqu'à 120000 casquettes en 1899) . $^{8}$ Qualité innée ou "qualification importée" ? Deux phénomènes se sont conjugués dans l'essor des immigrés dans l'industrie à Paris : déqualification pour bon nombre de tailleurs étrangers ; apprentissage sur place pour d'autres, qui n'avaient jamais manié une aiguille chez eux. Comme se lamentait Yankel Mykhanowitzki, juif russe à Paris, héros du roman Les Eaux mêlées de Roger Ikor : "Parfois il s'étonnait, assez naïvement, de s'ennuyer à l'atelier. Naguère encore, les moindres gestes du métier ne lui étaient-ils pas une source de plaisirs sans cesse renouvelés? Apprécier de loin la pièce de drap toute fraîche, goûter d'avance la souplesse de l'étoffe, son moelleux, cligner des yeux pour bien juger de la teinte. Un claquement de langue, un hochement de tête entendu, une moue de connaisseur: 'Hmm! Ça sera un bijou, je vous le dis !'... Non: cela, cétait autrefois, dans les temps rakwomiriens [de sa ville d'origine, Rakwomir]. Maintenant, Yankel travaillait en mécanique. Il ne plaisantait plus, ne souriait plus aux plaisanteries ${ }^{9}$."

Or, si certains ont apporté leur savoir-faire ou leurs modes avec eux, pour la grande majorité il s'agissait de trouver un travail rapidement et d'apprendre sur le tas. L'atelier de compatriotes, incarnation du réseau d'immigrés, fait fonction de lieu d'apprentissage comme lieu de socialisation et d'insertion dans le nouveau monde.

\section{L'univers de la confection et la main-d'œuvre étrangère}

Au début du XXe siècle, Käthe Schirmacher commentait : "L'immigration étrangère non qualifiée, venant de pays frustes, apporte son contingent de misère à cette industrie maudite: Slaves, Polonais et Russes surtout, israélites, Roumains et Turcs sont les plus exploités ${ }^{10}$." Elle notait d'abord les immigrés de la première vague, en provenance des pays frontaliers : 4220 Belges, 1885 Allemands, 1880 Italiens et 1425 Suisses, soit 9410 étrangers (sur 277755 travailleurs du vêtement).

Mais ces chiffres montrent déjà combien, si les immigrés sont disponibles pour la confection, la confection est une branche d'activité importante pour eux. Le vêtement constituait l'activité majeure (22\%) des immigrés belges ; il faisait vivre $10 \%$ des travailleurs allemands (après les secteurs de la domesticité et le commerce), 12,5 \% des Italiens (après le bâtiment et le commerce), et 9,8 \% des Suisses (après le commerce, y compris la banque, et la domesticité $^{11}$ ). Selon un guide yiddish Dans les années 1840, environ $40 \%$ des tailleurs parisiens étaient déjà étrangers, originaires d'Allemagne, de Hongrie et de Pologne, bien que la plupart de leurs ouvrières fussent françaises. pour les immigrants juifs publié en 1910, quelque 8860 juifs étaient employés dans la confection parisienne à l'époque, soit 29,3\% du total des travailleurs immigrés juifs de la capitale ${ }^{12}$. La structure du métier et les réseaux ne suffisent pas à eux seuls à expliquer comment les immigrés se sont installés à Paris comme à New York ou à Londres. Les flux migratoires sont eux-mêmes la conjugaison de conditions dans les pays d'origine poussant des milliers de personnes sur les chemins de l'exil et de conjonctures dans les pays d'attraction qui correspondent à autant de moments géopolitico-économiques. Le rôle des États n'est pas en reste, provoquant des départs ou accueillant des rescapés avec plus ou moins de bonne grâce ou de politiques d'immigration libérales (surtout en périodes d'expansion économique).

Quand les juifs fuyaient des pogroms de l'Empire tsariste, les Arméniens le génocide de 1915-1917,

8. Maurice Lauzel, Ouvriers juifs de Paris. Les casquettiers, Paris, Édouard Cornély et Ciel., 1912, p. 23 ; Léon Storch, Julien Hayem, Auguste Mortier, Exposition universelle internationale de 1900 à Paris. Rapports du jury international, Groupe XIII : Fils, tissus, vêtements, $2^{e}$ partie - Classes 85 et 86, Imprimerie nationale, 1902, p. 204-205. 9. Roger Ikor, Les Fils d’Avrom. Les Eaux mêlées, Paris, Albin Michel, 1955, pp. 159, 161. 10. Kathie Schirmacher, La Spécialisation du travail - Par nationalités, à Paris, Paris, Arthur Rousseau, 1908, pp. 71, 67, 92. Voir aussi Liliane Crips, "Comment passer du libéralisme au nationalisme volkish tout en restant féministe", in Marie-Claire Hoock-Demarle (dir.), Femmes, nations, Europe, Paris, université de Paris-VII, 1995. 11. Kathie Schirmacher, op. cit., pp. 15, 171-174. 12. Wolf Speiser, Kalendar, 1910, pp. 78-80. Conservé à la BNF : Yiddish-Franzoïsicher Kalendar 5671, cote m.9879. 
les Italiens la misère ou le fascisme, les juifs d'Afrique du Nord la décolonisation, les Arméniens le Liban, l'Iran ou la Turquie dans les années 1960 et 1970, les Sino-Khmers les Khmers rouges en 1975 et l'invasion vietnamienne du Cambodge en 1979, les Turcs (notamment les Kurdes qui s'expatrient d'une Turquie en pleine crise ou bien réfugiés politiques après le coup d'État de 1980), tout cela dessine un gigantesque kaléidoscope des peuples en marche. Tous ces conflits, sans parler

Il est impossible d'évaluer précisément l'impact de des aspirations individuelles, des ruptures familiales ont nourri les courants migratoires la Solution finale sur la maind'œuvre juive de la confection,

mais l'économiste Joseph

Klatzmann estime que

peut-être la moitié au moins des travailleurs juifs à domicile ont été déportés. des XIX et XX ${ }^{e}$ siècles. Ils sont allés là où les portes étaient ouvertes. Évidemment, tous n'ont pas choisila mode comme solution. L'histoire, la géographie et la politique expliquent que l'on a pu trouver, tout au long du XXe siècle, tant de juifs d'Europe de l'Est, d'Arméniens, de Turco-Kurdes, de Serbes, de juifs tunisiens, de Sino-khmers ou de Chinois de Chine dans les ateliers à Paris.

Le démographe Georges Mauco notait dans son ouvrage Les Étrangers en France, que le travail des étoffes était, après le commerce (autre activité d'entrée facile), l'activité professionnelle la plus importante pour les immigrés de Paris selon le recensement de 1926. Il y avait 4611 Polonais, 3115 Russes, 2629 Italiens, 2576 Belges, 1328 Roumains et 870 Arméniens/Grecs ${ }^{13}$. La Préfecture de police estimait en 1933 que sur les 27978 travailleurs étrangers de la confection du département de la Seine, 8462 étaient polonais, suivis par 5274 Italiens $^{14}$. On a estimé par ailleurs que $70 \%$ des ouvriers du vêtement pendant l'entre-deux-guerres étaient juifs (polonais et russes) $)^{15}$.

\section{Ouvriers juifs, ouvriers arméniens}

Les juifs d'Europe orientale n'étaient pourtant pas les seuls juifs du métier. Si après la Première Guerre mondiale, des juifs polonais se sont joints aux juifs russes d'avant 1914, des juifs turcs partis dans les ruines de l'Empire ottoman (comme des Arméniens rescapés du génocide) venaient également refaire leur vie en France ${ }^{16}$. Si l'entre-deux-guerres élargit l'éventail des origines des mécanicien(e)s de l'industrie de l'habillement, la Seconde Guerre mondiale a eu l'effet inverse. L'aryanisation des maisons juives et la déportation de 76000 juifs de France eurent des conséquences drastiques sur l'industrie. Il est impossible d'évaluer précisément l'impact de la Solution finale sur la main-d'œuvre juive de la confection, mais l'économiste Joseph Klatzmann estime que peut-être la moitié au moins des travailleurs juifs à domicile ont été déportés. Pourtant, dans les années sombres de l'Occupation et dans l'immédiat après-guerre, le caractère décentralisé et informel de la confection a également ménagé des possibilités de travail clandestin à domicile. Klatzmann a calculé qu'en 1946, les juifs représentaient $60 \%$ des ouvriers de sexe masculin de la confection parisienne. L'historien de Paris et des toponymies, Michel Roblin, quant à lui, considéra, au début des années 1950, que $40 \%$ des personnes de l'annuaire téléphonique de Paris regroupées sous la catégorie "confection" étaient juifs. Ce pourcentage montait à $60 \%$ pour les seuls casquettiers ${ }^{17}$.

La décolonisation des années 1950 et du début des années 1960 suscite de nouvelles vagues de travailleurs étrangers juifs bien que, dans un premier temps, ils sont plutôt petits commerçants avant de devenir fabricants à leur tour.

13. Georges Mauco, Les Étrangers en France, Paris, Armand Colin, 1932, p. 300. Voir aussi Élisabeth Rudinesco, "Georges Mauco (18991988). Un psychanalyste au service de Vichy. De l'antisémitisme à la psycho-pédagogie", in L'Infini, nº 51, automne 1995, pp. 69-84. 14. Cité dans L'Habillement, Paris, $n^{\circ}$ 16, 1939 ; Marie-Claude Blanc-Chaléard, “Immigration et implantation des Italiens dans l'espace parisien", in La Trace, $n^{\circ}$ 6, 1992, p. 23. 15. Passages, décembre 1988.16. Annie Benveniste, Le Bosphore à La Roquette. La communauté judéo-espagnole à Paris (1914-1940), Paris, L’Harmattan, 1989. 17. Joseph Klatzmann, Le Travail à domicile dans l'industrie parisienne du vêtement, Paris, Armand Colin, 1957, pp. 81-82, 86 ; Michel Roblin, Les Juifs de Paris, Paris, A. et J. Picard, 1952, pp. 99-100. Sur les casquettiers juifs, voir Maurice et Léon Bonneff, La Vie tragique des travailleurs [1908], Paris, EDI, 1981, pp. 260-263; Maurice Lauzel, op. cit.; Nancy L. Green, Les Travailleurs immigrés juifs à la Belle Époque, Paris, Fayard, 1985, pp.178-182, 207-221. 
Juifs tunisiens et marocains commencèrent par écouler des vêtements sur les marchés en tant que représentants ou grossistes, avant de se mettre à la fabrication, en profitant de la forte croissance économique des années 1960 et de l'essor du vêtement de loisir, le sportswear. Un délégué de la section du Sentier (IIe arrondissement de Paris) de la Fédération des juifs de France estimait au milieu des années 1980 que quelque 800 des 1200 firmes du quartier étaient juives, parmi lesquelles $90 \%$ appartenaient à des juifs d'Afrique du Nord (60 \% de Tunisie, 20 \% du Maroc, 10 \% d'Algérie), et $10 \%$ aux juifs d'Europe de l'Est ${ }^{18}$.

De la même manière, la communauté arménienne s'est renouvelée après-guerre. Nombre d'Arméniens, après une étape à Marseille ou à Lyon dans l'industrie de la soie, se sont installés à Paris ou plus exactement à Issy-les-Moulineaux au début des années 1920. Ce qui n'était au départ qu'un lieu de résidence devint dans les années 1940 un lieu d'activité professionnelle spécialisé dans le tricot. Les Arméniens de la deuxième génération qui restèrent dans le métier profitèrent eux aussi de l'expansion du sportswear dans les années 1960 et 1970, tandis qu'un nouveau groupe d'Arméniens arrivait en France au milieu des années 1970, venu de Turquie, du Liban et d'Iran ${ }^{19}$.

\section{Nouvelles vagues migratoires et luttes pour la régularisation}

Au gré des événements géopolitiques, la fin du XXe siècle apportera de nouvelles vagues en France et au Sentier. Des Serbes et des Turcs (souvent kurdes), ainsi qu'un grand nombre d'immigrants chinois (une minorité installée auparavant au Cambodge), ont profité de la croissance de cette industrie à Paris. Le gouvernement français signa des accords d'immigration avec la Turquie et la Yougoslavie en 1965 et 1966. À la fin des années 1970, de plus en plus de Yougoslaves (surtout des Serbes) choisirent Paris, le plus souvent dans des quartiers proches des maisons de confection de la rue du Faubourg-Saint-Martin ${ }^{20}$. Les Turcs vinrent également en France dans les années 1960, grâce à des accords bilatéraux. Les hommes arrivaient les premiers. Si certains avaient été tailleurs chez eux, la plupart venaient d'abord pour travailler dans les usines d'automobiles, le bâtiment, la fonderie ou l'agroalimentaire. Mais bientôt, les constructeurs d'automobiles licenciant leurs ouvriers en masse, beaucoup d'entre eux passèrent d'une industrie à une autre. Avec ou sans qualification, et souvent avec l'aide de leurs épouses et filles, ils Aujourd'hui, les délocalisations et les importations de vêtements à très bas prix ont suscité de nouvelles difficultés pour les fabricants français, tandis que de nouveaux immigrés sont en quête d'emplois. devenaient ouvriers de la confection, et quelquefois ouvraient leurs propres ateliers. À partir du début des années 1980, on vit ainsi apparaître des ateliers turcs dans les $\mathrm{X}^{\mathrm{e}}$ et $\mathrm{XI}^{\mathrm{e}}$ arrondissements, non loin du Sentier ${ }^{21}$.

En février 1980, 17 immigrés turcs sans papiers, soutenus par l'Association des travailleurs turcs, commencèrent une grève de la faim. Avec l'aide de la Confédération française démocratique du travail (CFDT), ils réclamaient la régularisation globale de leur situation, et non un traitement des dossiers au cas par cas. Ils obtinrent finalement gain de cause au moment des défilés de prêt-à-porter.

18. Or l'Institut national de la statistique et des études économiques (Insee) a compté 2700 établissements dans le quartier en 1986. Nancy L. Green et al., "Les quartiers parisiens de l'industrie de l'habillement et les relations pluri-ethniques", rapport, ministère de la Culture (MIRE), 1987, p. 107. 19. Martine Hovanessian, Lien communautaire. Trois générations d’Arméniens, Paris, L'Harmattan, 2007, voir surtout pp. 152-160, 208-213, 220-230; Nancy Green et al., "Les quartiers parisiens de l'industrie de l'habillement et les relations pluri-ethniques", op. cit., p.172 et chap. 6 (par Sandrine Tasmadjian) ; Nancy L. Green et al., "Les rapports habitat/travail dans l'industrie de l'habillement à Paris et dans sa banlieue", rapport, ministère de l'Équipement, du Logement, de l'Aménagement du territoire et des Transports, 1988, chap. 5 (par Sandrine Tasmadjian). 20. Solange Montagné-Villette, op. cit., p. 118 ; Mirjana Morokvasic, "Le recours aux immigrés dans la confection à Paris. Éléments de comparaison avec la ville de Berlin-Ouest", ministère du Travail et de la Formation professionnelle, Mission de liaison interministérielle pour la lutte contre les trafics de main-d'œuvre, 1986. 21. Kenan Ozturk, "Les Turcs dans la confection à Paris", in Hommes \& Migrations, $n^{\circ} 1116,1988$, pp. 22-28; Nancy L. Green et al., "Les rapports habitat/travail dans l'industrie de l'habillement à Paris et dans sa banlieue", op. cit., chap. 3 (par Kenan Ozturk); Solange Montagné-Villette, op .cit., pp. 117-118. 
En l'espace d'un an (au 31 janvier 1981), 2944 travailleurs du vêtement ont été régularisés. La liste de ces régularisations montre la variété des origines : 1389 Turcs (47,2 \% du total), 755 Yougoslaves (25,6 \%), 352 Marocains (12 \%), 151 Mauriciens (5,1\%), 102 Pakistanais (3,5 \%), 84 Tunisiens $(2,8 \%)$ et 111 "divers" (3,8\%). Les deux années suivantes, une régularisation plus massive intervint, pour quelque 83000 travailleurs immigrés, dont 9322 ouvriers de la confection ${ }^{22}$.

Les Chinois ne faisaient pas partie de ce premier groupe de travailleurs régularisés, car ils faisaient partie des 110000 réfugiés arrivant du Vietnam, du Cambodge et du Laos au milieu des années 1970, ont bénéficié de l'asile politique et n'avaient pas besoin de permis de travail. Ils n'ont pas été absents de l'industrie de l'habillement, loin s'en faut. À la fin du XXe siècle, d'autres immigrants chinois, originaires de la Chine populaire, participent à leur tour à la mobilisation pour leur régularisation en créant le Troisième collectif des sans-papiers ${ }^{23}$.

\section{Le renouvellement d'une industrie urbaine}

L'entrée dans le métier par les immigrés s'est donc faite historiquement à travers le travail à domicile ou en petits ateliers, et en tant que représentants ou grossistes. Mais une autre voie s'est ouverte pour des Pakistanais, des Sri-Lankais, des Mauriciens (généralement d'origine indienne) et des Africains (Sénégalais et Maliens) dans les dernières décennies du XXe siècle. Ils ont commencé à travailler dans le Sentier, le plus souvent comme débardeurs, livreurs ou porteurs. Vendant leur force de travail à la demi-journée, à la journée ou à l'heure, ils ont fait de la place du Caire un véritable tchowk (mot ourdou pour "lieu de rassemblement") au centre de Paris $^{24}$. En décembre 1982, les ouvriers pakistanais créèrent l'Association pour une solution au problème de l'emploi clandestin (Aspec) dans le Sentier avec le soutien de la CFDT, et en mars 1983, ils mirent sur pied une société coopérative de production (scop) de débardeurs pakistanais, qui faisait office d'entreprise de location de main-d'œuvre intérimaire. Mais les Pakistanais, de même que les Mauriciens (qui ont l'avantage de parler français) se sont eux aussi lancés dans la production et sont montés en grade, dans la coupe et la couture. Certains ont ouvert leur propre atelier ${ }^{25}$. Même le gargotier pakistanais qui proposait son curry a apparemment essayé l'assemblage de vêtements, tandis que Mehmood Bhatti devenait le premier patron pakistanais du Sentier. En période d'essor comme de déclin, cette industrie urbaine (l'une des dernières à résister dans les grandes métropoles, même si elle a fortement diminué) dépend des ouvriers étrangers qui acceptent, faute de mieux, d'être courbées de longues heures devant les machines à coudre et les bas salaires. Cette histoire ancienne s'explique donc : l'industrie a toujours eu besoin d'une main-d'œuvre flexible ; les immigrés ont besoin de travailler rapidement ; les seuils d'entrée en capital et en savoirfaire pour monter un atelier sont bas. Aujourd'hui, les délocalisations et les importations de vêtements à très bas prix ont suscité de nouvelles difficultés pour les fabricants français, tandis que de nouveaux immigrés sont en quête d'emplois. Lors des périodes de croissance comme de déclin, l'industrie du vêtement s'est révélée particulièrement adaptée aux besoins des fabricants comme des ouvriers à la recherche d'un travail. En fin de compte, l'industrie a servi aux immigrés et vice versa. Une chose est certaine : la mode "made in France" n'aurait pas pu se faire sans eux.

22. Rosine Klatzmann, Le Travail noir, Paris, PUF, 1982, pp. 29-30 ; Le Monde, 11 février 1981, 5-6 août 1984 ; Solange Montagné-Villette, op .cit., pp. 96, 117; Mirjana Morokvasic, op. cit. ; Riva Kastoryano, Être Turc en France. Réflexions sur famille et communauté, Paris, L'Harmattan, 1986, pp. 128-32. 23. Michelle Guillon, Isabel Taboada-Leonetti, Le Triangle de Choisy. Un quartier chinois à Paris, Paris, L'Harmattan, 1986, p. 133 ; Emmanuel Ma Mung, "Logiques du travail clandestin des Chinois", in Solange Montagné-Villette (dir.), Espaces et travail clandestins, Paris, Masson, 1991, pp. 99-106 ; Yinh Phong Tan, "Restaurants et ateliers. Le travail des sinokhmers à Paris”, in ASEMI, $n^{\circ}{ }_{15}, 1984$, pp. $277-291$; Véronique Poisson (dir.), “Chinois de France”, in Hommes \& Migrations, $n^{\circ} 1254$, mars-avril 2005. 24. Le Monde, 22 et 24-25 février 1983 ; 5-6 août 1984 ; 11-12 septembvre 1988 ; Nancy Green et al., op. cit., "Quartiers parisiens", pp. 108-113, 130-133 (par Annie Benveniste et Vasoodeven Vuddamalay); Solange Montagné-Villette, op. cit., pp. 118-120. 25. Vasoodeven Vuddamalay, "Les mécanismes de structuration du mouvement migratoire mauricien en France”, thèse à l'École des hautes études en sciences sociales, 1993, pp. 334-355. 